\title{
A Novel Pen-Based Calculator and Its Evaluation
}

\author{
William Thimbleby \\ now at Department of Computer Science \\ Swansea University, Singleton Park, Swansea, SA2 8PP \\ will@thimbleby.net
}

\begin{abstract}
A novel calculator, ideal for interactive whiteboards and pen-based devices, is introduced and evaluated. The calculator provides a natural, dynamic method of entering conventional expressions by handwriting and provides continual feedback showing the calculation and results. The user interface adjusts and copes with partial expressions, morphing the expressions to correct position and syntax. Gestures are also used to edit and manipulate calculations. The user interface is declarative, in that all displays, even with partial user input, are of correct calculations.

The new calculator is faster for more complex expressions and importantly, gives users more confidence in its use. The majority of users said that they would prefer to use this calculator rather than their conventional calculator.
\end{abstract}

\section{Author Keywords}

Handheld calculators, gesture input, novel interfaces.

\section{ACM Classification Keywords}

H5.2. Information interfaces and presentation (e.g., HCI): User Interfaces.

\section{INTRODUCTION}

Imagine writing a calculation down on paper, and the paper magically working out the answers. We have built a calculator that works like this, which is ideal for pen based user interfaces, or for interactive whiteboard use in classrooms. This paper discusses the design and its evaluation. (It will be demonstrated in the conference.)

\section{Overview}

Refer to Figure 1 at the top of the next page, which shows screen snapshots of the new calculator in use. We first show a user doing the sum $3+6$. In the first screen shot, they have written $3+6$, in the next the calculator is catching up with them and has already rendered the 3 and + in a printed font. In screenshot 3 the calculator has morphed the input into a

Paper presented at the ACM NordiCHI Conference 2004, the biannual Nordic $\mathrm{HCl}$ conference, held in Tampere, Finland. Cite as W. Thimbleby, "A Novel Pen-Based Calculator and Its Evaluation," Proceedings of the third Nordic conference on Humancomputer interaction, pp 445-448, 2004. nicely typeset equation. The user then clears the screen, screenshot 4, using a cross-out, $X$, gesture; the feedback from deletion, the 'smoke' feedback is also visible. The final two, 5 and 6 , screenshots show the user entering $2 / 3 x=4$, the declarative calculator ensures the answer is correct, and the interface morphs the answer into a nicely typeset and readable equation.

\section{History and Motivation}

We have always used instruments to aid our mental arithmetic and to help us with mathematics. Somewhere around 200AD, the abacus was invented, and in the 1970s with the development of electronics, electronic calculators became popular. For the most part their design copied earlier mechanical calculators. Now, thirty years later, when desktop and handheld computers can do almost anything, today's calculators merely imitate early electronic calculators. The calculator provided in the Start menu by Microsoft is less powerful, and less expressive, than a 10 year old handheld calculator! Yet computers today could do a lot better than just simple imitations of mechanical calculators.

The majority of current research on expression recognition has been directed towards that of expression entry $[1,2,3]$, although there have been attempts to marry expression entry with calculation (for example, the PenCalc project [4] Yet, none of the existing implementations have attempted to use expression recognition itself as a user interface for a calculator.

The calculator presented here, and its design extend the domain of calculator user interfaces into the $21^{\text {st }}$ century. Rather than relying on obsolete metaphors that dictate awkward and unnatural mathematical entry, this calculator provides a natural interface that is designed to (and does) function like pen and paper - or, rather, paper that does mathematics magically.

\section{PEN-BASED USER INTERFACES}

The main advantage of a pen-based system is the familiarity of the interface. The majority of users are already competent at writing with a pen. This advantage is greater with mathematical expressions, because the majority of mathematical work is still done on paper with a pen or pencil. Using a pen-based system to enter expressions is a natural progression, as it means that anyone can use it with little or no training. Pens can replicate the complete functionality of both keyboard and mouse, enabling computers with a sole input device. 

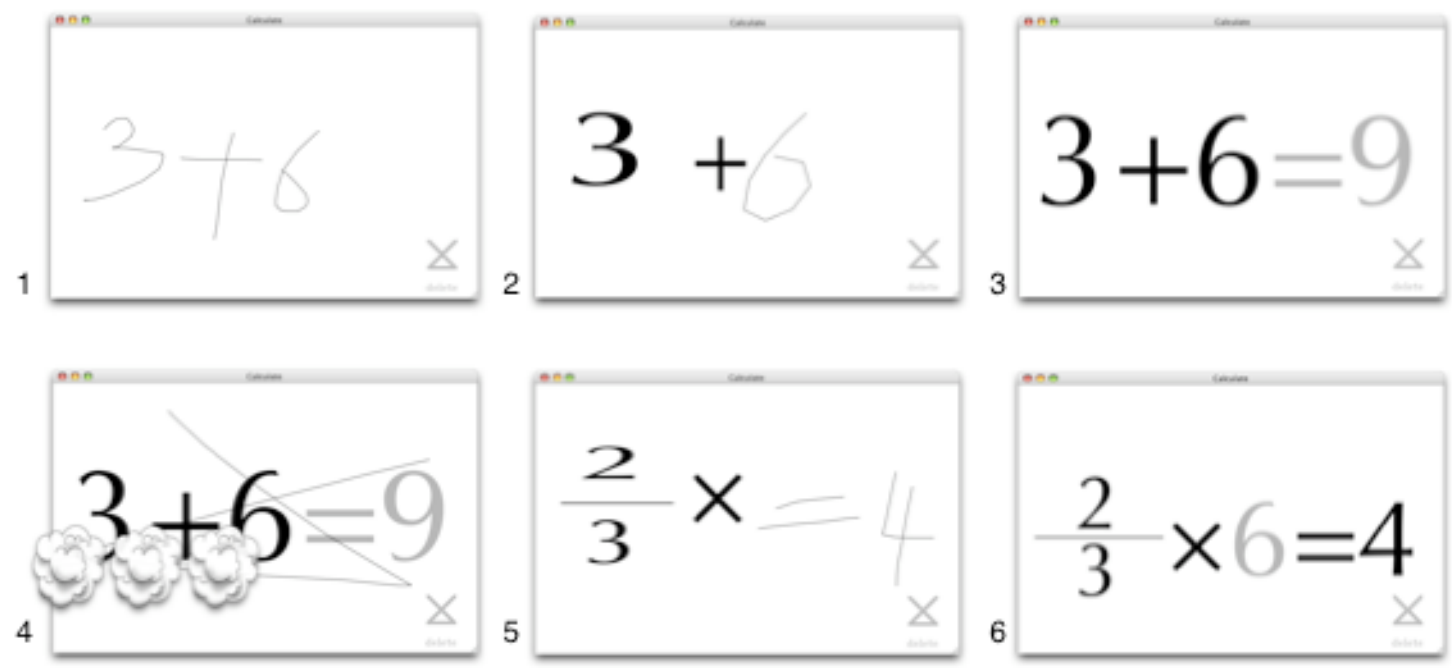

Figure 1. Screenshots of the calculator's progress computing, $3+6$ and then $2 / 3 \times 2=4$.

Meyer [6] gives a good detailed overview of the whole technology, including both a history of pen based computing and more technical aspects of the hardware and software.

\section{IMPLEMENTATION}

The implementation is written in Java, and is spilt into two modules. The first provides basic symbol recognition using a model-matching algorithm. The second recognises the equations using a recursive descent algorithm based on Chang [5]. The interaction and user-interface are layered on top of these two modules

\section{USER INTERFACE REQUIREMENTS}

Ideally a user interface for mathematical input should provide a superset of paper's functionality, allowing a user to use the interface in the exact way they would use paper. The key features of the paper metaphor are outlined below.

\section{Sketching}

Ideally a user could draw rough sketches. Often when solving a problem a user will not jump straight onto their computer and solve it, but jots down diagrams or figures first. The system presented does not implement sketching, however a solution is to specify areas for diagrams and mathematics.

\section{Expressions}

The system should allow the user to enter expressions as they would on paper, without unnatural restrictions. For instance, the user should not be forced to enter the expression in a strict fashion.

\section{Editing}

It should be possible to edit expressions at any time. Both input and output expressions (that is, an expression just entered, and one that has been computed) should be treated in the same manner. High-level editing, such as rearrang- ing, insertion and deletion should be possible. To implement these with a pen-based interface, without leaving the paradigm of pen and paper, requires special gestures that are assigned to each of the editing functions. For example, a scribble is used for deletion. Character editing is different and involves correcting the computer guess at the semantics of a set of strokes.

\section{Feedback}

The system should always keep the user informed about what is going on, providing appropriate timely feedback.

\section{THE DESIGN}

Our new calculator uses a single canvas for mathematical expressions, which enables us to create a completely modeless interface that is intuitive and natural. The user interface is shown in Figure 1. The operation of the user interface was developed from Thimbleby [7].

The one adornment of the interface is a delete gesture reminder in the bottom right corner.

\section{Expressions}

The system imposes small timing constraints. It requires the strokes of contiguous symbols to be written sequentially within a small amount of time. This allows the interface to recognise the user's input on-line as they enter it. These small restrictions were found to be unobtrusive, and not to affect the user's writing style.

\section{Editing}

After testing several different ways of editing expressions, it was found that for the majority of mathematical expressions, the easiest way to edit them was to delete and rewrite portions of the expression. This keeps the interface very simple. (Dragging or pop-up menus would create areas of the screen that function differently from each other.) By allowing only a simple delete gesture, no mode changes are 
necessary. Every part of the screen or virtual paper acts like paper: every click and drag draws.

\section{Feedback}

The visibility of the system's status is provided through two kinds of feedback: annotation and morphing. As the user is writing, the system can process in the background. As a symbol is recognised the user is informed of this recognition by visual feedback: a typeset character stretched to the stroke's bounding box replaces the written strokes.

Morphing starts after a short time delay from when the user stop writing. This halts when the user starts writing stopping it from distracting the user and from rearranging expressions as they are trying to enter them. The morph formats the entered expression into a correctly typeset equation by moving the symbols as little as possible from the user's writing. The morph provides continuity between the user's input and the typeset equation that allows them to continue to edit and use it.

\section{EVALUATION}

A total of nine participants, 5 students and 4 members of the public, took part in the usability testing. These ranged in ability from a mathematics student to people who rarely use mathematics. The testing comprised of a number of mathematics questions based on old GCSE papers. Users were given time to familiarize themselves with the interface. When they were happy, they were observed and recorded whilst attempting to complete the questions using the new system and their own pocket calculator or one provided (a Sharp EL-531GH DAL).

An observer was present and users were encouraged to discuss problems with them, afterwards the users filled in a short anonymous questionnaire.

Measurements were recorded of the time taken and the number of errors or problems encountered entering expressions. The questionnaires provided a better general impression of the ease of use.

\section{RESULTS}

\section{Time on Task}

For the simpler sums, like $9 \times 2 / 3$, a handheld calculator was much faster than the new system. An average of 24 seconds for the new system compared to 10 seconds for the traditional handheld calculator. This was expected. The majority of users were familiar with handheld calculators, and had used them over many years.

Two of the tasks were actually faster on the new system. Calculating Figure 2, was faster (an average of $49 \mathrm{~s}$ to $79 \mathrm{~s}$ ) because users could enter it "as they saw it" rather than having to search for buttons on a calculator.

$$
\frac{2^{2}}{21-4}
$$

Figure 2. A simple equation.

For the task "What power of two is 28?" every user was able to complete the task on the new system, yet most struggled to solve it on a handheld calculator. Solving it is easy, in a similar way to Figure 1 picture 6, using the novel declarative approach from [7].

Thus this new calculator enables users to perform mathematics that they could not do before. Furthermore, it is faster for more complicated expressions because users did not have to rearrange the expression in their head. This was the not even the case for those who knew the rearrangement $\log 28 \div \log 2$.

\section{Accuracy}

A large part of the time taken to complete the tasks was taken up with recovering from symbol recognition errors. Currently the accuracy percentage in this prototype (81.1\%) is poor, but easily improved. This significantly lowers the usability of the system. Expression recognition caused only a very few errors, mostly caused by short divisor bars.

However, when calculating mathematics, input accuracy is not the most important consideration; output accuracy is. No user got the wrong answer for any question with the new system. In contrast several unnoticed mistakes were made with the traditional calculators.

By displaying the computed equation in an easily understandable two-dimensional format, it provided the feedback necessary to understand what was being computed. Thus users knew when their calculations were wrong with the new calculator.

\section{Ease of Use}

The overall impression from users was that the new calculator was easy to use. Typesetting and feedback though morphing successfully allowed the user to understand what the calculator was doing.

No user had trouble editing expressions using the delete gesture. Other editing functions like cut and paste were never missed and users liked the modeless interface and the simplicity of one function.

\section{Fun}

Several users wanted to carry on playing with the system and asked when they could get their own copy.

\section{FURTHER WORK}

During both testing and design many ideas were developed that provide possible avenues for further development. The more interesting are outlined here. 


\section{Extended Vocabulary}

Expanding the number of symbols recognised to include symbols like $\pi$, letters, and other Greek characters, would enable the system to handle more complex expressions.

Additionally, extra functional vocabulary would allow the system more power and expressiveness. For instance trigonometric functions, user defined constants, logarithms, and factorials.

\section{The User Interface}

Further additional features of paper (for instance, sketching) would add to the usability.

Users specifically requested two additional features. A clear button clears the whole screen, a similar metaphor to starting a new page. This could be provided as a simple gesture or an external button.

Secondly, users found that there sometimes was not enough room to enter their additional symbols into an existing expression. Two solutions for further work would be, the addition of an insert space gesture that adds in a gap into an expression and the re-morphing of an expression as a user writes to accommodate the user's input.

\section{CONCLUSION}

At its most abstract, this paper described a novel pen based interface for any application. This paper described and evaluated the pen-based interface for a dynamic, on-line mathematical calculator.

New user interface concepts for the computation of mathematics were introduced, including:

- The combination of pen user interface with an on-line calculator.

- Extra space added to calculations, like longer division bars, to aid the addition of more symbols.

- The use of a single delete gesture to edit expressions, making the calculator completely modeless and providing the user with an extremely simple interface.

The comparative user testing comparing the new system with traditional calculators showed that:

- Answers produced by the new calculator were more accurate. In contrast, users failed to notice when a traditional calculator gave them a wrong answer - errors that they noticed when using the new calculator.

- Users were able to calculate the answer to problems using the new calculator that they could not solve using traditional calculators.

- Users are able to obtain accurate answers and have greater confidence in those answers compared to results from traditional calculators.
- A pen based calculator is more intuitive, fun, and easy to use than traditional calculators.

- The pen is a suitable device for entering and editing mathematical expressions. Additionally, more complex editing operations than delete are neither necessary nor missed.

- For complex calculations, the new design was faster than using traditional calculators.

It is hoped that the creation of this new calculator will prompt people to rethink the methods by which we do mathematics. (The calculator is available on the web for others to build on.) Calculators are currently restricted by obsolete metaphors, as the testing and creation of this new calculator has shown.

Ultimately, the calculator should be ported to and tested on pen based, handheld computers and tablet PCs, as well as in school classrooms (e.g., using projectors and touchsensitive whiteboards) where they would be an ideal way of teaching mathematics to children.

We are confident that the prototype described in this paper charts a course in the right direction.

\section{AVAILABILITY}

A movie of the calculator and the Java application are available at http://www.uclic.ucl.ac.uk/usr/will/

\section{REFERENCES}

1. Dorothea Blostein and Andy Schüerr. Computing with graphs and graph transformation. Software Practice and Experience, 29(3):1-21, 1999.

2. Yuko Eto and Masakazu Suzuki. Mathematical formula recognition using virtual link network. In Proceedings of the Sixth International Conference on Document Analysis and Recognition (ICDAR '01), page 762. IEEE Computer Society, 2001.

3. Steve Smithies. Freehand formula entry system. Master's thesis, University of Otago, Dunedin, New Zealand, May 1999.

4. Pencalc http://www.cs.ust.hk/pencalc/.

5. S. Chang. A method for the structural analysis of twodimensional mathematical expressions. Information Sciences, 2(3):253-272, 1970.

6. A. Meyer. Pen computing: a technology overview and a vision. SIGCHI Bulletin, 27(3):46-90, 1995.

7. H. Thimbleby. A new calculator and why it is necessary. The Computer Journal 38(6):418-433, 1995. 